\title{
Insulin response to carbohydrate ingestion after gastric surgery with special reference to hypoglycaemia
}

\author{
A. J. CAMERON, J. P. Ellis, J. I. McGill, AND L. P. Le QUESNe \\ From the Department of Surgical Studies and The Institute of Nuclear Medicine, \\ The Middlesex Hospital, London
}

\begin{abstract}
SUMMARY Factors responsible for spontaneous hypoglycaemia were investigated in 23 gastrectomy patients and two patients with vagotomy and pyloroplasty. Plasma insulin and capillary blood glucose levels were followed after giving $175 \mathrm{ml}$ of $50 \%$ glucose orally. In gastrectomy patients a significant correlation was found between the height of the peak blood glucose and insulin levels for the same individual. Patients with high peak glucose and insulin levels were significantly more likely to develop hypoglycaemia later in the test. These findings are compatible with the suggestion that the major factor predisposing to hypoglycaemia is a faster than average rate of emptying of the gastric remnant, but this does not explain all the results. In two cases, hypoglycaemia followed an abnormally large insulin response to oral glucose. The results of insulin injection tests in 14 patients do not exclude the further possibility that in some cases hypoglycaemia is due to excessive insulin sensitivity.
\end{abstract}

Symptomatic hypoglycaemia may occur one and a half to three hours after meals in patients who have undergone partial gastrectomy (Adlersberg and Hammerschlag, 1947; Zollinger and Hoerr, 1947) or vagotomy with a drainage procedure (Tanner, 1966). Only a minority of patients have hypoglycaemic attacks, however, and the reason for this is uncertain. The development of an immunoassay technique (Yalow and Berson, 1960) has made it possible to estimate plasma insulin levels in large numbers of samples. The object of the present study was to use this technique to investigate the difference between those patients with and those without hypoglycaemic symptoms after gastric surgery.

\section{MATERIAL AND METHODS}

Twenty-three patients who had undergone partial gastrectomy for benign peptic ulcer were studied (Table). Four of these had also had a truncal vagotomy. In addition, two patients who had had vagotomy with pyloroplasty were investigated, their results being discussed separately from the main group. Ten patients gave a history suggesting spontaneous hypoglycaemia. Twelve gastrectomy patients were studied as outpatients, the remainder being admitted to hospital, usually for investigation of postgastrectomy problems. The patients studied, while not a random sample, represented a spectrum from excellent to bad gastrectomy results. Patients were studied without special dietary preparation. The average daily intake of dietary calories and carbohydrate was estimated for outpatients by a dietician and was measured for those admitted to hospital. Tests were performed in the morning, patients fasting overnight except that outpatients were allowed a sugar-free drink before leaving home. Patients rested sitting for $\mathbf{3 0}$ minutes before tests began.

For the oral glucose test, the patient while seated drank $175 \mathrm{ml}$ of $50 \%$ glucose rapidly, this being the amount given in earlier studies from this department (Le Quesne, Hobsley, and Hand, 1960). Symptoms were recorded during the test. Blood was drawn at approximately 30-minute intervals for three and a half hours, capillary blood being taken five minutes after venous sampling.

For the insulin sensitivity test, $\mathbf{0 . 0 6}$ unit of soluble insulin per $\mathrm{kg}$ body weight was given intravenously and capillary blood taken at 15-minute intervals for one hour.

Blood glucose was measured by the glucose-oxidase method (Huggett and Nixon, 1957). At the height of glucose absorption, the difference between simultaneous capillary and venous levels was found to vary between 10 and $130 \mathrm{mg} / 100 \mathrm{ml}$, as previously noted by Mosenthal and Barry (1950). Capillary blood, taken by finger prick after warming the hand, was therefore used for glucose estimations, since this gives a more accurate reflection of the arterial level than venous blood (Jonas, 1933). Plasma insulin was measured on heparinized venous blood by the immunoassay method of Hales and Randle (1963a) using iodinated insulin $\left({ }^{131} \mathrm{I}\right){ }^{1}$ Duplicate estimations were

${ }^{1}$ Radiochemical Centre, Amersham. 
TABLE

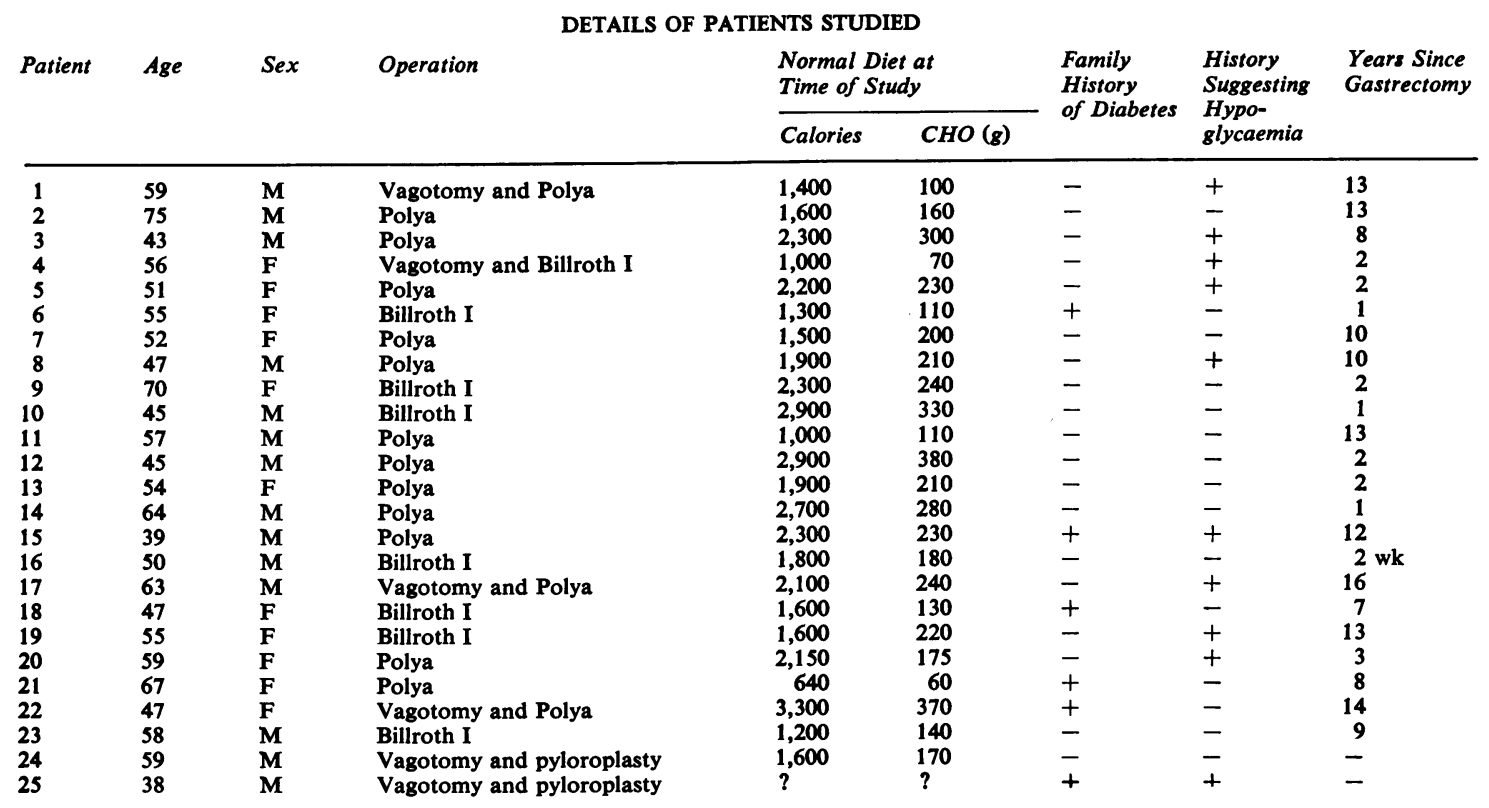

performed on at least two separate assays for every sample.

\section{RESULTS}

Most oral glucose tolerance curves showed a high early peak and then a sharp fall, which sometimes continued down to a hypoglycaemic level (Fig. 1). The peak glucose level was reached between 35 and 65 minutes. In three patients the minimum glucose level occurred at 95 minutes, in six at 125 minutes, in seven at 155 minutes, in six at 185 minutes, and in one patient at 215 minutes.

All fasting plasma insulin levels were $20 \mu \mathrm{U} / \mathrm{ml}$ or less. After glucose ingestion, insulin levels usually rose steeply, sometimes following an initial delay. Thereafter, changes in the insulin level closely paralleled changes in the blood glucose. When the blood glucose concentration was lowest the insulin level was at, or rapidly approaching, the fasting level. One gastrectomy patient showed an unusually large insulin response (Fig. 2a). Those with low peak glucose levels had low peak insulin levels and were less likely to have a low blood glucose in the later part of the test (Fig. 2b). Taking the gastrectomy patients as a whole, three significant correlations were found: $(a)$ the higher the peak glucose level, the higher the peak insulin level (Fig. 3); (b) the higher the peak glucose level, the lower the subsequent minimum glucose level (Fig. 4); and (c) the higher the peak insulin level, the lower the minimum glucose level (Fig. 4). The four patients with both vagotomy and gastrectomy had high peak glucose and insulin levels, but their results did not suggest that vagotomy altered the insulin response to a given glucose level, or altered their sensitivity to endogenous insulin (Figs. 3 and 4). Results in the two patients with vagotomy and pyloroplasty only are shown in Figure 5. One showed a very large, and the other a rather small, insulin response, both being within the range observed in gastrectomy patients.

No correlation was found between the mean daily carbohydrate intake of the $\mathbf{2 3}$ gastrectomy patients and either the peak glucose level $(r=0.0621)$, the peak insulin level $(r=0.0611)$, or the minimum glucose level $(r=0 \cdot 1636)$ on their oral glucose tests.

Many patients had dumping symptoms of varying severity within the first hour after glucose ingestion but hypoglycaemia occurred later after a symptomfree interval. Hypoglycaemic symptoms were reproduced in four of the nine gastrectomy patients and in the one patient with vagotomy and pyloroplasty, who gave a history suggesting spontaneous attacks. One of the 15 patients with no previous history developed hypoglycaemia during the test. The capillary glucose levels of these six patients at the time of hypoglycaemic symptoms were $28,30,36,42,49$, and $60 \mathrm{mg} / 100 \mathrm{ml}$. (Venous glucose levels at that time were a mean of $13 \mathrm{mg} / 100 \mathrm{ml}$ lower.) Observed attacks lasted about 10 to 20 minutes and symptoms included a feeling of undue warmth, sweating, shakiness, dizziness, and difficulty in concentration, 


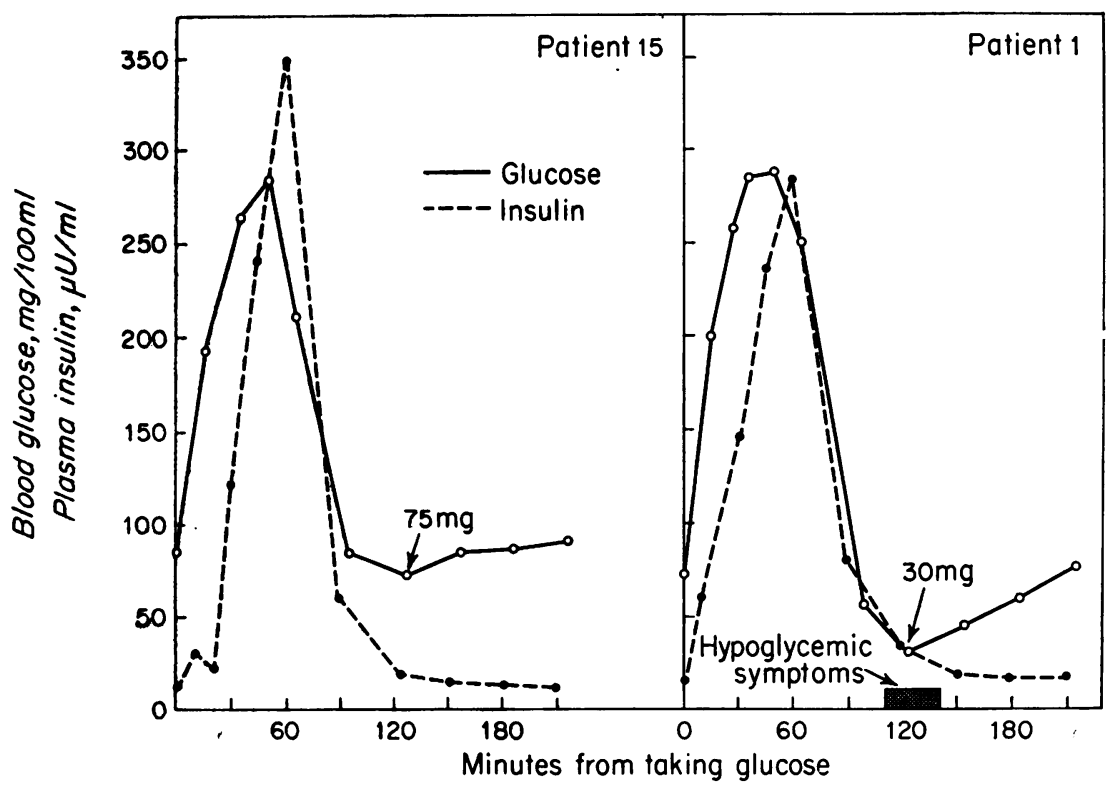

FIG. 1a and b. Glucose and insulin levels in two gastrectomy patients after ingestion of $175 \mathrm{ml} 50 \%$ glucose.

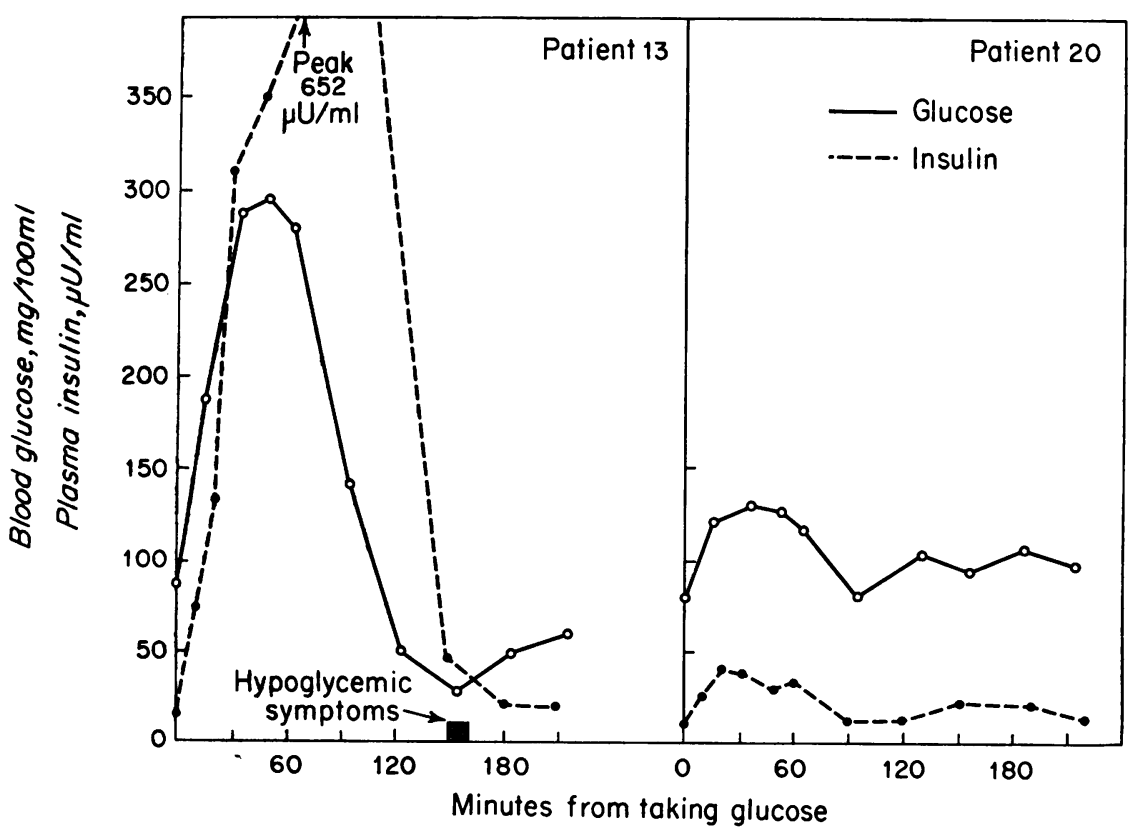

FIG. 2. Glucose and insulin levels in two gastrectomy patients after ingestion of $175 \mathrm{ml} 50 \%$ glucose showing (a) an unusually large insulin response and (b) low peak glucose and insulin levels. 


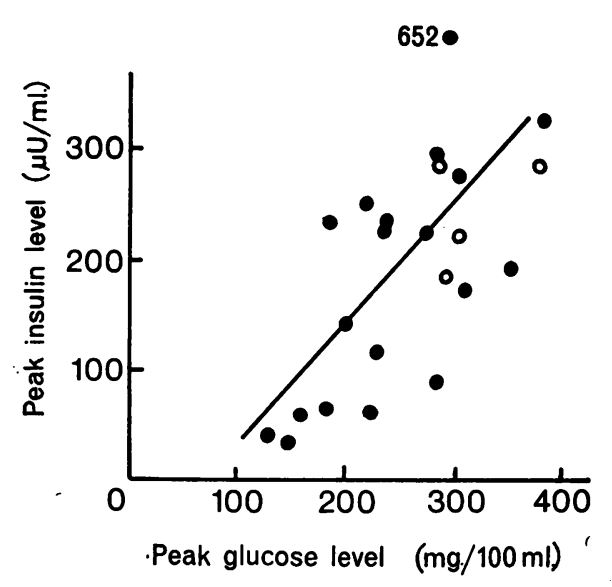

FIG. 3. Correlation between peak glucose and insulin levels in gastrectomy patients after glucose ingestion. $\mathrm{O}=$ patients after vagotomy and gastrectomy. $\mathrm{r}=0.559$, $\mathrm{P}=<0.005, y=1.07-70 x$.

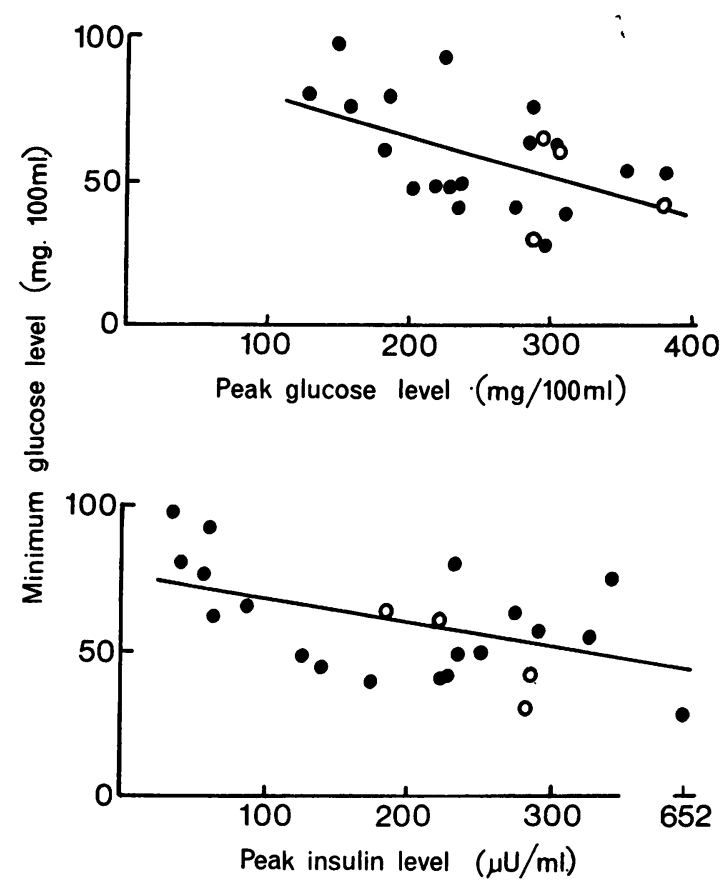

FIG. 4. Above, correlation between early peak glucose and late minimum glucose levels in gastrectomy patients after glucose ingestion. $\mathrm{O}=$ vagotomy and gastrectomy. $\mathrm{r}=$ $0.5025, \mathrm{P}=<0.01, y=93-0.133 x$.

Below, correlation between early peak insultn and late minimum glucose levels in gastrectomy patients after oral glucose. $O=$ vagotomy and gastrectomy. $\mathrm{r}=-0.5836$, $\mathrm{P}=<0.005, y=75-0.0809 x$.

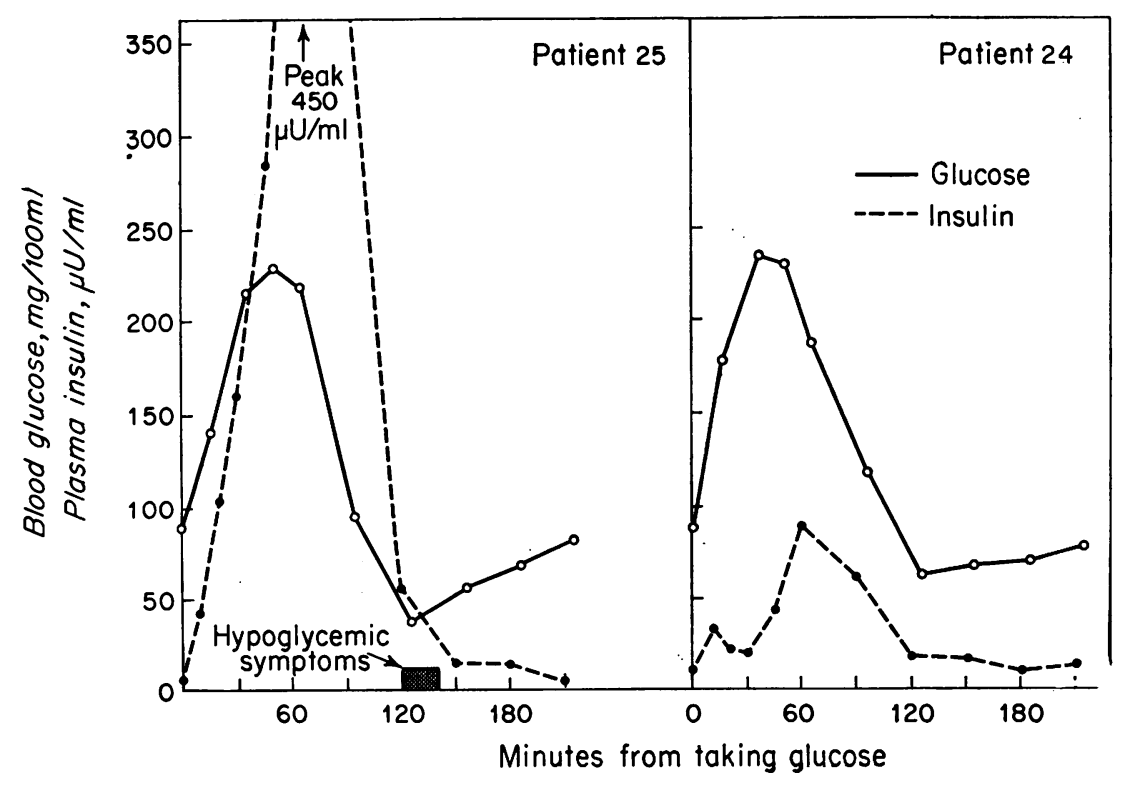

FIG. 5a and b. Glucose and insulin levels in two patients with vagotomy and pyloroplasty showing different insulin responses associated with similar early peak blood glucose levels. 


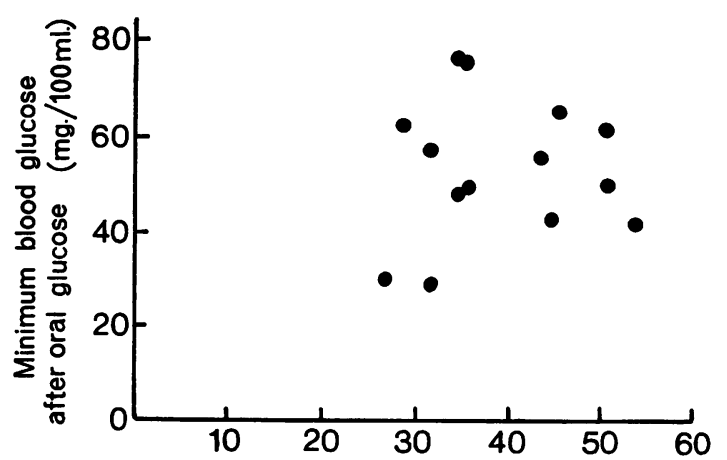

Minimum blood glucose after i.v. insulin ( $\mathrm{ng} / 100 \mathrm{ml}$ )

FIG. 6. Minimum glucose levels after oral glucose and after intravenous insulin in the same 14 gastrectomy patients. $\mathrm{r}=0.3704, \mathrm{P}=>0.1$.

no patient becoming confused or comatose.

Fourteen gastrectomy patients had insulin sensitivity tests (Fig. 6). There was poor correlation between the minimum glucose level after oral glucose and after insulin for the same patient $(r=0.3704$, $P>0 \cdot 1)$ suggesting that differing sensitivity to insulin was not an important factor in determining the occurrence of hypoglycaemia after carbohydrate ingestion.

Gastric remnant emptying was studied in two additional patients given $175 \mathrm{ml}$ of $50 \%$ glucose after positioning a nasogastric tube fluoroscopically. At 35 minutes their blood glucose levels were 214 and $263 \mathrm{mg} / 100 \mathrm{ml}$ and aspiration five minutes later recovered 41 and $13 \mathrm{~g}$ glucose respectively.

\section{DISCUSSION}

There is some general agreement as to the cause of postgastrectomy hypoglycaemia. The gastric remnant empties faster than the intact stomach (Bruusgaard, 1946) so that ingested carbohydrate is rapidly presented to and absorbed from the small intestine. As a result, peak blood glucose and insulin levels are higher in gastrectomy patients than in normal subjects (Phear, 1962; Roth and Meade, 1965). Excessive insulin release in response to hyperglycaemia is thought to cause subsequent hypoglycaemia (Muir, 1949; Conn and Seltzer, 1955).

Gastrectomy patients given intravenous glucose rarely develop hypoglycaemia (Butler, 1951) probably because the insulin response to intravenous glucose is less than if glucose is absorbed from the intestine (McIntyre, Holdsworth, and Turner, 1964; Elrick, Stimmler, Hlad, and Arai, 1964). However, in the oral glucose tests in the present study, changes in the plasma insulin level closely followed changes in the blood glucose level suggesting that the latter was the principal factor determining the insulin response.

Variations in the rate and pattern of gastric emptying offer one explanation why only a few gastrectomy patients develop hypoglycaemia. In the oral glucose tests in the present study, patients whose peak glucose and insulin levels were highest were most liable to develop hypoglycaemia later in the test. A rapid initial rate of gastric emptying may cause a higher glucose peak before secreted insulin takes effect. Smith, Fraser, Staynes, and Willcox (1953) found no difference in the emptying rate of a barium-glucose meal between gastrectomy patients with and without hypoglycaemic attacks. However, radiological assessment of gastric emptying is only semi-quantitative, and due to its high specific gravity, barium may pass rapidly through a dependent gastrectomy stoma and so may not accurately reflect the emptying rate of food or a glucose test meal. Gastric emptying tests on two of our patients confirmed that the gastric remnant has considerable reservoir function and suggested that the emptying rate of hypertonic glucose can vary considerably. Also, although we have no direct evidence for this, the tail end of a meal is perhaps sometimes still leaving the gastric remnant at two to three hours, thus protecting against hypoglycaemia. This could explain the difference in minimum glucose levels between the patients whose tests are illustrated in Figure 1.

An unusually large insulin response to oral glucose was seen in two patients developing hypoglycaemia during the test, one with a gastrectomy (no. 13) and one with a vagotomy and pyloroplasty (no. 25). The first patient had never had such symptoms before, whereas the other had frequent, troublesome attacks. This suggests that hypoglycaemia is sometimes due to an excessive insulin response to a given blood glucose level, but that this may not be a consistent occurrence in the same individual.

In our patients with the combined operation of vagotomy and partial gastrectomy the insulin response to a given blood glucose level and the hypoglycaemic effect of endogenous insulin were unremarkable. The higher incidence of hypoglycaemic symptoms reported after vagotomy with a drainage procedure as compared with partial gastrectomy (Goligher, Pulvertaft, De Dombal, Conyers, Duthie, Feather, Latchmore, Shoesmith, Smiddy, and Willson-Pepper, 1968) are perhaps related to faster gastric emptying after vagotomy (McGill, Cameron, Hobsley, and Le Quesne, 1968) rather than to any direct effect of vagotomy on insulin release or action.

Increased sensitivity to exogenous insulin could not be demonstrated statistically in our gastrectomy 
patients developing hypoglycaemia after oral glucose. However, the two patients with the lowest minimum glucose levels after oral glucose were more sensitive than most to injected insulin. Barnes (1947) suggested that postgastrectomy hypoglycaemia might be due to increased insulin sensitivity, but Muir (1949) found increased sensitivity to injected insulin in only one of 12 patients with hypoglycaemia after oral glucose. 'Smith et al (1953) found a delay in recovery from hypoglycaemia after insulin injection in patients with a history of hypoglycaemic attacks, although the mean minimum glucose level reached was not as low as in gastrectomy patients without attacks. We are inclined to agree with Muir (1949) that increased insulin sensitivity cannot be eliminated as a factor predisposing to hypoglycaemia in some cases.

On a low carbohydrate diet, normal subjects show impaired oral glucose tolerance, reduced sensitivity to injected insulin (Himsworth, 1935), and a smaller plasma insulin response to oral glucose (Hales and Randle, 1963b). Many of our patients could not tolerate a full carbohydrate diet because of dumping symptoms and tests were therefore performed without special dietary preparation. No correlation was found between the dietary carbohydrate content and either the peak insulin level or the peak or minimum glucose levels after oral glucose. The previous diet was not, therefore, a significant factor in determining the shape of the glucose tolerance curves in these gastrectomy patients. It was noted that patients with a history of hypoglycaemia included both those who had restricted food intake due to fear of dumping attacks, and those who were able to eat heartily.

Hypoglycaemia occurring several hours post prandially may be due to pre-diabetes (Seltzer, Fajans, and Conn, 1956). However, a family history of diabetes, which would increase the chance of the patient having pre-diabetes, was found about as frequently in those with as those without hypoglycaemia in the present series.

We wish to thank Miss Margaret Phipps and Mr Geoffrey Paice for valuable technical assistance. We would like to record our gratitude to the many patients who volunteered to help in our studies.

\section{REFERENCES}

Adlersberg, D., and Hammerschlag, E. (1947). The postgastrectomy syndrome. Surgery, 21, 720-729.

Barnes, C. G. (1947). Hypoglycaemia following partial gastrectomy. Lancet, 2, 536-539.

Bruusgaard, C. (1946). The operative treatment of gastric and duodenal ulcer. Acta chir. scand., suppl. 117, 154.

Butler, T. J. (1951). A study of the significance of reactive hypoglycemia following gastrectomy. Gastroenterology, 19, 99-112.

Conn, J. W., and Seltzer, H. S. (1955). Spontaneous hypoglycemia. Amer. J. Med., 19, 460-478.

Elrick, H., Stimmler, L., Hlad, C. J., Jr, and Arai, Y. (1964). Plasma insulin response to oral and intravenous glucose administration. J. clin. Endocr., 24, 1076-1082.

Goligher, J. C., Pulvertaft, C. N., De Dombal, F. T., Conyers, J. H., Duthie, H. L., Feather, D. B., Latchmore, A. J. C., Shoesmith, J. H., Smiddy, F. G., and Willson-Pepper, J. (1968). Five-toeight-year results of Leeds/York controlled trial of elective surgery for duodenal ulcer. Brit. med. J., 2, 781-787.

Hales, C. N., and Randle, P. J. (1963a). Immunoassay of insulin with insulin-antibody precipitate. Biochem. J., 88, 137-146.

,$--(1963 b)$. Effects of low-carbohydrate diet and diabetes mellitus on plasma concentrations of glucose, non-esterified fatty acid, and insulin during oral glucose-tolerance tests. Lancet, 1, 790-794.

Himsworth, H. P. (1935). The dietetic factor determining the glucose tolerance and sensitivity to insulin of healthy men. Clin. Sci., 2, 67-94.

Huggett, A. St. G., and Nixon, D. A. (1957). Use of glucose oxidase, peroxidase, and o-dianisidine in determination of blood and urinary glucose. Lancet, 2, 368-370.

Jonas, L. (1933). A note on cutaneous venous blood sugar difference in normal males and females and in thyroid disease. J. clin. Invest., 12, 139-141.

Le Quesne, L. P., Hobsley, M., and Hand, B. H. (1960). The dumping syndrome. I. Factors responsible for the symptoms. Brit. med. J., 1, 141-147.

McGill, J. I., Cameron, A. I., Hobsley, M., and Le Quesne, L. P. (1968). The effect of fat upon gastric emptying after partial gastrectomy and after vagotomy with a drainage procedure. (Abstr.) Brit. J. Surg., 55, 386.

McIntyre, N., Holdsworth, C. D., and Turner, D. S. (1964). New interpretation of oral glucose tolerance. Lancet, 2, 20-21.

Mosenthal, H. O., and Barry, E. (1950). Criteria for and interpretation of normal glucose tolerance tests. Ann. intern. Med., 33, 1175-1194.

Muir, A. (1949). Postgastrectomy syndromes. Brit. J. Surg., 37, 165-178.

Phear, D. N. (1962). The normal and diabetic patterns of insulin response to glucose. Lancet, 2, 955-958.

Roth, D. A., and Meade, R. C. (1965). Hyperinsulinism-hypoglycemia in the postgastrectomy patient. Diabetes, 14, 526-528.

Seltzer, H. S., Fajans, S. S., and Conn, J. W. (1956). Spontaneous hypoglycemia as early manifestation of diabetes mellitus. Ibid., $5,437-442$.

Smith, W. H., Fraser, R., Staynes, K., and Willcox, J. M. (1953). The causes of post-prandial attacks of palpitation and weakness after gastric operation. Quart. J. Med., 22, 381-404.

Tanner, N. C. (1966). Disabilities which may follow the peptic ulcer operation. Proc. roy. Soc. Med., 59, 362-368.

Yalow, R. S., and Berson, S. A. (1960). Immunoassay of endogenous plasma insulin in man. J. clin. Invest., 89, 1157-1175.

Zollinger, R. M., and Hoerr, S. O. (1947). Gastric operations. Troublesome postoperative symptoms with special reference to carbohydrate ingestion. J. Amer. med. Ass., 134, 575-579. 